\title{
A new approach to the theory of electromagnetic interactions with bound systems: calculations of deuteron magnetic and quadrupole moments
}

\author{
Alexandr Shebeko* \\ Institute for Theoretical Physics National Scientific Center "Kharkov Institute of Physics \& \\ Technology", Kharkov 61108, Ukraine \\ E-mail: shebekodkipt.kharkov.ua
}

Ivgen Dubovyk

Institute of Electrophysics \& Technologies, Kharkov 61002, Ukraine

E-mail: e.a.dubovykemail.ru

\begin{abstract}
The method of unitary clothing transformations in quantum field theory is applied for describing electromagnetic interactions with nuclei (bound systems). As before, while studying the pion electroproduction off deuterons and radiative capture of light nuclei at astrophysical energies, the corresponding reaction amplitudes are represented in an explicitly gauge independent form starting from the Nöther current operator for interacting meson and nucleon fields and using the well-known trick after Foldy. In the framework of the field-theoretic approach we propose fresh prescriptions to evaluate the electric and magnetic Cartesian moments of the system. As a byproduct, the electric quadrupole and magnetic dipole moments of deuterons are calculated relying on our recent explorations of the nucleon-nucleon $(N-N)$ scattering below the pion production threshold.
\end{abstract}

XXI International Baldin Seminar on High Energy Physics Problems September 10-15, 2012

JINR, Dubna, Russia

\footnotetext{
* Speaker.
} 
To the memory of M. I. Shirokov

Excellent Scientist and

Modest Person

\section{Preamble}

A field-theoretic approach based on the method of unitary clothing transformations (UCTs) exposed in refs. [四], [四], [B] and [䧃] is applied in the theory of electromagnetic interactions (EM) with nuclei. In this context, we will show the clothed particle representation (CPR) not only of the original field Hamiltonian and other generators of the Poincare group but the current density operator too. In accordance with the gauge invariance principle (GIP) (see, e.g., [四], [可]) the latter must meet the continuity equation (CE) that allows one to derive important results (in particular, the so-called low-energy theorems) for the EM transitions. One of them will be employed below when finding links between the quantum-mechanical definitions of the static EM moments of nuclei (bound systems) and properties of the photonuclear reaction amplitudes at small momentum transfers. An explicitly gauge independent (GI) form [可], [四] of the EM transition amplitudes for single-photon absorption or emission on a nucleus makes possible to get new prescriptions when evaluating its Cartesian moments.

As an illustration, we consider the $e-d$ scattering to see how the notion of clothed particles can be incorporated into the theory of EM processes on nuclei. To be constructive our calculations of the simplest deuteron moments are carried out with the deuteron state (the bound state of the clothed neutron and proton) that is an eigenvector of the corresponding Hamiltonian in the CPR, whose interaction part has been built up in [8] starting from a conventional model (see, e.g., [Q]) for interacting $\pi-, \rho-, \omega-, \sigma$ - and $N(\bar{N})$-fields. Therefore, the $n-p$ interaction operator consists of separable contributions due to exchanges of the clothed mesons between the clothed nucleons. Following [ [8], in order to avoid ultraviolet divergences typical of local field theories we prefer to handle the regularized contributions to the interaction Hamiltonian density by introducing some covariant cutoff functions in momentum space. Our consideration is compatible with relativistic invariance requirements being fulfilled in the framework of an original procedure in [ए0] to meet the Poincaré-Lie algebra.

Further, in spite of the primary field model used has the same departure point as that by the Bonn group $[Q]$ there are appreciable distinctions between the UCT and Bonn potentials that ensure a fair treatment of such on-energy-shell quantities as the phase shifts of the $n-p$ scattering (cf. the dotted and solid curves in Figs.7 and 8 in [ [8]]). These distinctions become especially prominent in case of the ${ }^{3} S_{1}-{ }^{3} D_{1}$ potentials responsible for the formation of the tensor part of nuclear forces. One more motivation of our current explorations is search for quantities essentially dependent on their properties and the first candidate to this direction may be the $S$ - and $D$-components of the deuteron wave function (WF). In this connection, of great interest are the electric quadrupole and magnetic dipole moments of deuterons. The topic has an eventful history. But here let us remind of the relativistic calculations within: the light-front dynamics [ㅁ] ] and [ㅁ], the Bethe-Salpeter formalism [[12]] and [ㅍ], the inclusion of meson exchange currents (MEC) and boost contributions of leading order [ए5] and [ए]6] and refs. therein. 


\section{Electromagnetic Static Moments of Bound Systems}

Starting from the operator

$$
\vec{\mu}=\frac{1}{2} \int d \vec{x} \vec{x} \times \vec{J}(\vec{x})
$$

of the magnetic dipole moment for a system with the current density $\vec{J}(\vec{x})$ (reminescent of the BiotSavart formula from the classical magnetostatics) one can show after [ㅁ]] that its matrix elements between narrow wave packets are expressed through the limit

$$
\lim _{\vec{q} \rightarrow 0}\left[-\frac{i}{2} \operatorname{curl}_{\vec{q}}\left\langle\frac{\vec{q}}{2}|\vec{J}(0)|-\frac{\vec{q}}{2}\right\rangle\right]
$$

where the matrix elements $\langle\vec{q} / 2|\vec{J}(0)|-\vec{q} / 2\rangle^{1}$ determine the corresponding current in the Breit frame. The magnetic dipole moment of the system, being defined as the $z$-component of the vector (‥]) for the stretched configuration with $M_{J}=J$, in case of the deuteron

$$
\mu_{d}=\lim _{\vec{q} \rightarrow 0}\left[-\frac{i}{2} \operatorname{curl}_{\vec{q}}\left|\frac{\vec{q}}{2} ; 1\right| \vec{J}(0)\left|-\frac{\vec{q}}{2} ; 1\right\rangle\right]^{z} .
$$

In parallel, considering the interaction energy of the system, which has the charge density $\rho(\vec{x})=$ $J^{0}(\vec{x})$, in a static external electric field and expanding it in the Cartesian electric moments one encounters the quadrupole moment tensor

$$
Q_{i j}=\int d \vec{x}\left[3 x_{i} x_{j}-\delta_{i j} \vec{x}^{2}\right] \rho(\vec{x}) \quad(i, j=1(x), 2(y), 3(z)) .
$$

Then, repeating the same trick with the wave packets one gets the matrix elements

$$
\left\langle J M_{J}^{\prime}\left|Q_{i j}\right| J M_{J}\right\rangle=-\lim _{\vec{q} \rightarrow 0}\left[\left\{3 \frac{\partial^{2}}{\partial q_{i} \partial q_{j}}-\delta_{i j} \frac{\partial^{2}}{\partial q_{l}^{2}}\right\}\left\langle\frac{\vec{q}}{2}|\rho(0)|-\frac{\vec{q}}{2}\right\rangle\right] .
$$

to introduce the electric quadrupole moment $Q=\left\langle J J\left|Q_{33}\right| J J\right\rangle$ (again, not necessarily for the deuteron). At the point, let us a little deviation.

\section{Two Forms of Electron-Deuteron Scattering Amplitude}

To retain the GI treatment for inevitably approximate calculations of the amplitudes of onephoton radiative processes we prefer to employ a generalization LevShe93 of the Siegert theorem

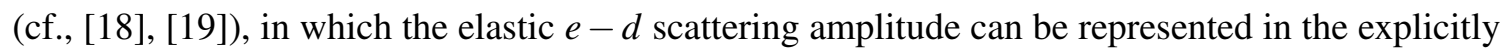
GI form:

$$
T\left(e d \rightarrow e^{\prime} d^{\prime}\right)=\left[\omega \vec{\varepsilon}\left(e^{\prime}, e\right)-\vec{q} \varepsilon_{0}\left(e^{\prime}, e\right)\right] \vec{D}(\vec{q})+\left[\vec{q} \times \vec{\varepsilon}\left(e^{\prime}, e\right)\right] \vec{M}(\vec{q}),
$$

with the generalized electric dipole moment

$$
\vec{D}(\vec{q})=-i \omega^{-1} \int_{0}^{1} \frac{d \lambda}{\lambda} \nabla_{\vec{q}}\left\{\left[\sqrt{\lambda^{2} \vec{q}^{2}+m_{d}^{2}}-m_{d}\right]\left\langle\lambda \vec{q} ; M^{\prime}|\rho(0)| \overrightarrow{0} ; M\right\rangle\right\}
$$

\footnotetext{
${ }^{1}$ The total angular moment $J$, its projection $M_{J}$ and other quantum numbers, if any, are implied
} 
and the generalized magnetic dipole moment

$$
\vec{M}(\vec{q})=-i \int_{0}^{1} d \lambda \nabla_{\vec{q}} \times\left\langle\lambda \vec{q} ; M^{\prime}|\vec{J}(0)| \overrightarrow{0} ; M\right\rangle,
$$

Here we have introduced the notation $\varepsilon_{\mu}\left(e, e^{\prime}\right)=\bar{u}_{e^{\prime}}\left(k^{\prime}\right) \gamma_{\mu} u_{e}(k)$ omitting the electron polarization labels. As usually, the Dirac spinor $u_{e}(k)\left(u_{e^{\prime}}\left(k^{\prime}\right)\right)$ describes the incident (outgoing) electron with the 4-momentum $k\left(k^{\prime}\right)$ and $q_{\mu}=(\omega, \vec{q})=k_{\mu}-k_{\mu}^{\prime}\left(q_{\mu}^{2}=\omega^{2}-\vec{q}^{2}<0\right)$ is the 4-momentum transfer. These equations have been derived using the property of translational invariance

$$
J^{\mu}(\vec{x})=e^{-i \vec{P} \vec{x}} J^{\mu}(0) e^{i \vec{P} \vec{x}}
$$

and the Foldy representation [[20] for

$$
\vec{a} \mathrm{e}^{i \vec{b} \vec{c}}=\int_{0}^{1}\left\{\nabla_{\vec{c}}\left(\vec{a} \cdot \vec{c} \mathrm{e}^{i \lambda \vec{b} \vec{c}}\right)+i \lambda \vec{c} \times[\vec{a} \times \vec{b}] \mathrm{e}^{i \lambda \vec{b} \vec{c}}\right\} \mathrm{d} \lambda
$$

with arbitrary vectors $\vec{a}, \vec{b}$ and $\vec{c}$.

We consider eq. (B.D) as an alternative to other (perhaps, more popular) form

$$
T\left(e d \rightarrow e^{\prime} d^{\prime}\right)=\varepsilon_{\mu}\left(e, e^{\prime}\right)\left\langle\vec{P} ; M^{\prime}\left|J^{\mu}(0)\right| \overrightarrow{0} ; M\right\rangle,
$$

where the deuteron current is expressed through the charge monopole $\left(G_{C}\right)$, magnetic dipole $\left(G_{M}\right)$ and electric quadrupole $\left(G_{Q}\right)$ form factors (FFs) of the deuteron (see survey [2]] and refs. therein), e.g. in lab. system with the Z-axis directed along the momentum transfer $\vec{q}$,

$$
\begin{gathered}
\left\langle\vec{q} ; M^{\prime}\left|J^{0}(0)\right| \overrightarrow{0} ; M\right\rangle=e(1+\eta)\left\{G_{C}\left(Q^{2}\right)+2 \eta\left[\delta_{M 0}-\frac{1}{3}\right] G_{Q}\left(Q^{2}\right)\right\} \delta_{M^{\prime} M} \\
\left\langle\vec{q} ; M^{\prime}\left|J^{x}(0)\right| \overrightarrow{0} ; M\right\rangle=\frac{e}{\sqrt{2}} \sqrt{\eta(\eta+1)} G_{M}\left(Q^{2}\right)\left\{\delta_{M^{\prime}, M+1}-\delta_{M^{\prime}, M-1}\right\}
\end{gathered}
$$

with ratio $\eta=\frac{Q^{2}}{4 m_{d}^{2}}>0$.

Static quantities such as the deuteron charge $e_{d}$, moments $\mu_{d}$ and $Q_{d}$ are given by normalization conditions

$$
e_{d}=e G_{C}(0), \mu_{d}=G_{M}(0) \frac{e}{2 m_{d}}, Q_{d}=G_{Q}(0) \frac{e}{m_{d}^{2}}
$$

One should note that the parametrization of the deuteron current in terms of three (no more) covariant FFs becomes possible once the current, first, meets the GI condition

$$
q_{\mu}\left\langle\vec{q} ; M^{\prime}\left|J^{\mu}(0)\right| \overrightarrow{0} ; M\right\rangle=0
$$

and, second, has the property

$$
U(\Lambda) J^{\mu}(0) U^{\dagger}(\Lambda)=J^{v}(0) \Lambda_{v}^{\mu}
$$

to be the 4-vector. By definition, the correspondence $\Lambda \Rightarrow U(\Lambda) \forall \Lambda$ that belong to the full Lorentz group (including the space inversion and time reversal) realizes its irreducible representation in the 
Hilbert space $\mathscr{H}$.

Evidently, eq. (B.JI) does not follow merely from the CE that is

$$
\left[P_{\mu}, J^{\mu}(0)\right]=0,
$$

this $e^{1}$-order consequence of the GIP whose historical roots go back to the never-to-be-forgotten realm created by Fock and Weyl (details in [[2]). Simultaneous ensuring of requirements (B.TU) and (B.12) is a nontrivial problem in practical calculations.

Thus, one has to handle the matrix elements of the Nöther operator $J^{\mu}(x)=\left(J^{0}(x) \equiv \rho(x), \vec{J}(x)\right)$ at the space-time point $x=(t, \vec{x})=0$, sandwiched between the initial $\overrightarrow{0} ; M\rangle$ (in the rest frame) and final $\left|\vec{P}=\vec{q} ; M^{\prime}\right\rangle$ deuteron states, and we will show finding of them within the CPR.

\section{Hamiltonian and Boost Generator for Meson-Nucleon System in CPR. Deuteron Eigenvalue Equation}

Recall that the CPR of the primary Hamiltonian $H \equiv H(\alpha)=H_{F}(\alpha)+H_{I}(\alpha)$ and other operators (for instance, in the case of interacting meson and nucleon fields) being expressed through the set $\alpha$ of bare-particles creation/annihilation operators is constructed via the UCT $W(\alpha)=W\left(\alpha_{c}\right)=$ $\exp \left[R\left(\alpha_{c}\right)\right]\left(R^{\dagger}=-R\right)$ that does the transition $\alpha=W\left(\alpha_{c}\right) \alpha_{c} W^{\dagger}\left(\alpha_{c}\right)$ to the new set $\alpha_{c}$ of clothedparticles creation/annihilation operators. Its generator $R$ is determined in such a way to remove from $H$ by means of the similarity transformation

$$
H(\alpha)=W\left(\alpha_{c}\right) H\left(\alpha_{c}\right) W^{\dagger}\left(\alpha_{c}\right)
$$

the so-called bad terms that prevent the bare vacuum and the bare one-particle state to be the $H$

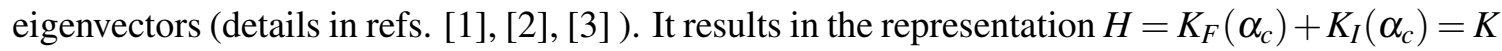
desired, where the free part $K_{F}\left(\alpha_{c}\right)=H_{F}\left(\alpha_{c}\right)$ while operator $K_{I}\left(\alpha_{c}\right)$ contains interactions between the clothed mesons and nucleons and destroys the physical vacuum $\Omega$ (the lowest-energy $H$ eigenstate) and the clothed one-particle states.

Doing so with the conventional scalar (s), pseudoscalar (ps) and vector (v) meson-nucleon couplings determined by formulae (3)-(5) in [ [8] we encounter the separate four-operator contributions of class [2.2],

$$
\begin{aligned}
& K_{I}^{(2)}\left(\alpha_{c}\right)=K(N N \rightarrow N N)+K(\bar{N} \bar{N} \rightarrow \bar{N} \bar{N})+K(N \bar{N} \rightarrow N \bar{N})+ \\
& K(b N \rightarrow b N)+K(b \bar{N} \rightarrow b \bar{N})+K\left(b b^{\prime} \rightarrow N \bar{N}\right)+K\left(N \bar{N} \rightarrow b b^{\prime}\right),
\end{aligned}
$$

responsible for the different $2 \rightleftarrows 2$ processes. In particular, the $N$-N interaction operator can be written as $K(N N \rightarrow N N)=\sum_{b} K_{b}(N N \rightarrow N N) \equiv K_{N N}$,

$$
\begin{aligned}
K_{b}(N N & \rightarrow N N)=\int \sum_{\mu} d \vec{p}_{1}^{\prime} d \vec{p}_{2}^{\prime} d \vec{p}_{1} d \vec{p}_{2} V_{b}\left(1^{\prime}, 2^{\prime} ; 1,2\right) \\
& \times b_{c}^{\dagger}\left(1^{\prime}\right) b_{c}^{\dagger}\left(2^{\prime}\right) b_{c}(1) b_{c}(2) \sim b_{c}^{\dagger} b_{c}^{\dagger} b_{c} b_{c},
\end{aligned}
$$

where symbol $\sum_{\mu}$ denotes summation over nucleon spin projections, $1=\left\{\vec{p}_{1}, \mu_{1}\right\}$, etc. Analytic expressions for the c-number matrices $V_{b}$ are given by eqs. (19)-(22) in [8]. Unlike [ $[8]$, where 
the UCT method has been applied when describing the $N-N$ scattering below the pion production threshold, let us consider the eigenvalue equation

$$
\left[H_{F}(\alpha)+H_{I}(\alpha)\right]\left|\Psi_{d}(\vec{P})\right\rangle=E_{d}\left|\Psi_{d}(\vec{P})\right\rangle
$$

or in the CPR

$$
\left[K_{F}(\alpha)+K_{I}\left(\alpha_{c}\right)\right]\left|\Psi_{d}(\vec{P})\right\rangle=E_{d}\left|\Psi_{d}(\vec{P})\right\rangle,
$$

with the energy $E_{d}=\sqrt{m_{d}^{2}+\vec{P}^{2}}, m_{d}=m_{p}+m_{n}-\varepsilon_{d}$ and deuteron binding energy $\varepsilon_{d}$. In the approximation with $K_{I}\left(\alpha_{c}\right)=K_{N N}$ we arrive to

$$
\left[K_{N}+K_{N N}\right]|\vec{P} ; M\rangle=E_{d}|\vec{P} ; M\rangle
$$

in the subspace $\mathscr{H}_{2 N} \subset \mathscr{H}$ spanned onto the basis $b_{c}^{\dagger} b_{c}^{\dagger}|\Omega\rangle$ with $K_{N} \sim b_{c}^{\dagger} b_{c}$ and $K_{N N} \sim b_{c}^{\dagger} b_{c}^{\dagger} b_{c} b_{c}$, where

$$
|\vec{P} ; M\rangle=\int d \vec{p}_{1} d \vec{p}_{2} D_{M}\left(\vec{P} ; \vec{p}_{1} \mu_{1}, \vec{p}_{2} \mu_{2}\right) b_{c}^{\dagger}\left(\vec{p}_{1} \mu_{1}\right) b_{c}^{\dagger}\left(\vec{p}_{2} \mu_{2}\right)|\Omega\rangle,
$$

with the coefficients $D_{M}\left(\vec{P} ; \vec{p}_{1} \mu_{1}, \vec{p}_{2} \mu_{2}\right)=\delta\left(\vec{P}-\vec{p}_{1}-\vec{p}_{2}\right) \psi_{M}\left(\vec{p}_{1} \mu_{1}, \vec{p}_{2} \mu_{2}\right), \psi_{M}(1,2)=-\psi_{M}(2,1)$.

For our aims it suffices to consider the eigenvalue problem in the deuteron rest frame,

$$
\begin{gathered}
\left|\psi_{M}\right\rangle=\left[m_{d}-K_{N}\right]^{-1} K_{N N}\left|\psi_{M}\right\rangle, \\
\left|\psi_{M}\right\rangle \equiv|\vec{P}=0 ; M\rangle=\int d \vec{p} \psi_{M}\left(\vec{p} \mu_{1},-\vec{p} \mu_{2}\right) b_{c}^{\dagger}\left(\vec{p} \mu_{1}\right) b_{c}^{\dagger}\left(-\vec{p} \mu_{2}\right)|\Omega\rangle .
\end{gathered}
$$

In a moving frame the corresponding eigenvector can be determined either by solving eq. (4.6) or using

$$
|\vec{P} ; M\rangle=\exp \left[-i \vec{\beta} \vec{B}\left(\alpha_{c}\right)\right]\left|\psi_{M}\right\rangle
$$

In the CPR the boost operator $\vec{B}\left(\alpha_{c}\right) \equiv W\left(\alpha_{c}\right) \vec{N}\left(\alpha_{c}\right) W^{\dagger}\left(\alpha_{c}\right)=\vec{B}\left(\alpha_{c}\right)=\vec{B}_{F}\left(\alpha_{c}\right)+\vec{B}_{I}\left(\alpha_{c}\right)$ consists of the free $\vec{B}_{F}$ and interaction $\vec{B}_{I}$ parts. Here $\vec{N}=\vec{N}_{F}+\vec{N}_{I}$ is the entire boost operator for the interacting fields in the instant form of relativistic dynamics employed. Relevant expressions can be found in [ᄄ口] ]. Perhaps, one should note that the required $\hat{P}^{\mu}|\vec{P} ; M\rangle=P^{\mu}|\vec{P} ; M\rangle$ follows from the property of the energy-momentum operator $\hat{P}^{\mu}=\left(H, \hat{P}^{1}, \hat{P}^{2}, \hat{P}^{3}\right)$ to be the four-vector, viz.,

$$
\begin{gathered}
e^{-i \vec{\beta} \vec{B}} \hat{P}^{\mu} e^{i \vec{\beta} \vec{B}}=\hat{P}^{v} L_{v}^{\mu}(\vec{\beta}), \\
L(\vec{\beta})=\left[\begin{array}{ccc}
L_{0}^{0}=P^{0} / m_{d} & \vdots & L_{0}^{j}=P^{j} / m_{d} \\
\cdots & \cdots & \cdots \\
L_{i}^{0}=P_{i} / m_{d} & \vdots & L_{i}^{j}=\delta_{i}^{j}-\frac{P_{i} P^{j}}{m_{d}\left(P^{0}+m_{d}\right)}
\end{array}\right]
\end{gathered}
$$

of the Lorentz transformation $m_{d}(1,0,0,0) \Rightarrow\left(P^{0}, P^{1}, P^{2}, P^{3}\right)=P,\left(\beta^{1}, \beta^{2}, \beta^{3}\right)=\vec{\beta}$ are related to velocity $\vec{v}=\vec{P} / m_{d}$ of the moving frame $\vec{\beta}=\beta \vec{n}, \vec{n}=\vec{v} / v, \tanh \beta=v$. In order to meet eq. (4.丁I) it is sufficient to meet the two Lie-Poincaré commutations

$$
[K, \vec{B}]=i \vec{P},\left[P_{i}, B_{j}\right]=i \delta_{i j} K
$$


To the approximation that leads to $K_{I}=K_{N N}$ the operator $B_{I} \sim b_{c}^{\dagger} b_{c}^{\dagger} b_{c} b_{c}$.

Using the basis vectors $\left|p(l S) J M_{J}, T M_{T}\right\rangle$ introduced in [8] we obtain

$$
\begin{gathered}
\left|\psi_{M, 00}\right\rangle \equiv\left|\psi_{M}\right\rangle=\frac{1}{\sqrt{2}} \sum_{l=0,2} \int_{0}^{\infty} p^{2} d p|p(l 1) 1 M\rangle \psi_{l}(p) \\
\psi_{M}\left(\vec{p} \mu_{1} \tau_{1},-\vec{p} \mu_{2} \tau_{2}\right)=\frac{1}{\sqrt{2}} \sum \psi_{l}(p) Y_{l m_{l}}(\hat{\vec{p}})\left(\operatorname{lm}_{l} 1 M_{S} \mid 1 M\right)\left(\frac{1}{2} \mu_{1} \frac{1}{2} \mu_{2} \mid S M_{S}\right)\left(\frac{1}{2} \tau_{1} \frac{1}{2} \tau_{2} \mid 00\right) .
\end{gathered}
$$

We accept the normalization $\left\langle\psi_{M^{\prime}} \mid \psi_{M}\right\rangle=\delta_{M^{\prime} M}$ which is equivalent to

$$
\int_{0}^{\infty} p^{2} d p\left[\psi_{0}^{2}(p)+\psi_{2}^{2}(p)\right]=1
$$

The "radial" components $\psi_{l}(p)(l=0,2)$ meet the set of homogeneous integral equations

$$
\psi_{l}(p)=\frac{1}{m_{d}-2 E_{\vec{p}}} \sum_{l^{\prime}} \int_{0}^{\infty} k^{2} d k V_{l l^{\prime}}^{J=S=1, T=0}(p, k) \psi_{l^{\prime}}(k) .
$$

\section{Current Density Operator in CPR}

We consider the expansion in the $R$-commutators

$$
J^{\mu}(0)=W J_{c}^{\mu}(0) W^{\dagger}=J_{c}^{\mu}(0)+\left[R, J_{c}^{\mu}(0)\right]+\frac{1}{2}\left[R,\left[R, J_{c}^{\mu}(0)\right]\right]+\ldots
$$

where $J_{c}^{\mu}(0)$ is the initial current in which the bare operators $\{\alpha\}$ are replaced by the clothed ones $\left\{\alpha_{c}\right\}$. Decomposition (لل involves the one-body, two-body and more complicated interaction currents. In its turn, the operator (5. Deing between the clothed two-nucleon states contributes as $^{2}$

$$
\eta_{c} J^{\mu}(0) \eta_{c}=J_{o n e-b o d y}^{\mu}+J_{t w o-b o d y}^{\mu}
$$

where the operator

$$
J_{\text {one-body }}^{\mu}=\int d \vec{p}^{\prime} d \vec{p} F_{p, n}^{\mu}\left(\vec{p}^{\prime}, \vec{p}\right) b_{c}^{\dagger}(\vec{p}) b_{c}(\vec{p})
$$

with

$$
F_{p, n}^{\mu}\left(\vec{p}^{\prime}, \vec{p}\right)=e \bar{u}\left(\vec{p}^{\prime}\right)\left\{F_{1}^{p, n}\left[\left(p^{\prime}-p\right)^{2}\right] \gamma^{\mu}+i \sigma^{\mu v}\left(p^{\prime}-p\right)_{v} F_{2}^{p, n}\left[\left(p^{\prime}-p\right)^{2}\right]\right\} u(\vec{p})
$$

that describes the virtual photon interaction with the clothed proton (neutron).

The formula (5.3) follows from the observation for which the primary Nöther current operator, being inserted between the physical (clothed) states $\left|\Psi_{N}\right\rangle=b_{c}^{\dagger}|\Omega\rangle$, yields the usual on-mass-shell expression

$$
\left\langle\Psi_{p, n}\left(\vec{p}^{\prime}\right)\left|J^{\mu}(0)\right| \Psi_{p, n}(\vec{p})\right\rangle=F_{p, n}^{\mu}\left(\vec{p}^{\prime}, \vec{p}\right)
$$

\footnotetext{
${ }^{2}$ Here $\eta_{c}$ is the projection operator on $\mathscr{H}_{2 N}$
} 
in terms of the Dirac and Pauli nucleon FFs.

By keeping in the r.h.s. of Eq. (5.2) only the one-body contribution we arrive to certain off-energyshell extrapolation of the so-called relativistic impulse approximation (RIA) in the theory of e.m. interactions with nuclei (bound systems). Of course, the RIA results should be corrected including more complex mechanisms of the $e-d$ scattering, that are contained in

$$
J_{t w o-b o d y}^{\mu}=\int d \vec{p}_{1}^{\prime} d \vec{p}_{2}^{\prime} d \vec{p}_{1} d \vec{p}_{2} F_{M E C}^{\mu}\left(\vec{p}_{1}^{\prime}, \vec{p}_{2}^{\prime} ; \vec{p}_{1}, \vec{p}_{2}\right) b_{c}^{\dagger}\left(\vec{p}_{1}^{\prime}\right) b_{c}^{\dagger}\left(\vec{p}_{1}^{\prime}\right) b_{c}\left(\vec{p}_{1}\right) b_{c}\left(\vec{p}_{2}\right) .
$$

They are immediately associated with the two-body MECs if one uses the terminology adopted in the theory of EM interactions with nuclei (see [23]). Analytic expressions for the coefficients $F_{M E C}^{\mu}$ stem from the $R$-commutators (starting with the third one) in the expansion ([ل]), which, first, belong to the class [2.2], as in Eq. (5.6), and, second, depend on even numbers of mesons involved. Their finding is a separate task that leads to a new (off-shell) family of the MECs.

\section{Deuteron Static Moments}

Further, with help of the relation

$$
e^{-i \vec{\beta}\left(\frac{\vec{q}}{2}\right) \vec{B}} \vec{J}^{l}(0) e^{i \vec{\beta}\left(\frac{\vec{q}}{2}\right) \vec{B}}=J^{\mu}(0) L_{\mu}^{l}\left(-\vec{\beta}\left(\frac{\vec{q}}{2}\right)\right)
$$

one can see that

$$
\mu_{d}=M^{z}(\vec{q}=0)=\frac{1}{2 m_{d}}\left\langle\overrightarrow{0} ; 1\left|[\vec{B} \times \vec{J}(0)]^{z}\right| \overrightarrow{0} ; 1\right\rangle
$$

and we will show our calculations in the approximation, where $\vec{B} \Rightarrow \vec{B}_{F}$ and $\vec{J}(0) \Rightarrow \vec{J}_{\text {one-body }}$. The former is given by

$$
\begin{aligned}
& \vec{B}_{F}=\vec{B}_{n u c l}=\sum d \vec{p}^{\prime} d \vec{p} \vec{B}_{\tau}\left(\vec{p}^{\prime} \mu^{\prime}, \vec{p} \mu\right) b_{c}^{\dagger}\left(\vec{p}^{\prime} \mu^{\prime} \tau\right) b_{c}(\vec{p} \mu \tau), \\
& \vec{B}_{\tau}\left(\vec{p}^{\prime} \mu^{\prime}, \vec{p} \mu\right)=i \frac{m}{4} \frac{E_{\vec{p}^{\prime}}+E_{\vec{p}}}{\sqrt{E_{\vec{p}^{\prime}} E_{\vec{p}}}} u^{\dagger}\left(\vec{p}^{\prime} \mu^{\prime}\right) u(\vec{p} \mu)\left[\frac{\partial}{\partial \vec{p}^{\prime}}-\frac{\partial}{\partial \vec{p}}\right] \delta\left(\vec{p}^{\prime}-\vec{p}\right) .
\end{aligned}
$$

while the latter by (5.3]) - (5.4) with $F_{\tau}^{v}\left(\vec{p}^{\prime} \mu^{\prime}, \vec{p} \mu\right)=e p^{v} / E_{\vec{p}} F_{1}^{\tau}(0) \delta_{\mu^{\prime} \mu}, F_{1}^{p}(0)=1, F_{1}^{n}(0)=0$, $2 m F_{2}^{p}(0)=\mu_{p}-1=1.793,2 m F_{2}^{n}(0)=\mu_{n}=-1.913$.

Now all we need is

i) to calculate vacuum expectation $\left\langle\Omega\left|b_{c} b_{c} b_{c}^{\dagger} b_{c} b_{c}^{\dagger} b_{c} b_{c}^{\dagger} b_{c}^{\dagger}\right| \Omega\right\rangle$ getting

$$
\begin{gathered}
\left\langle\overrightarrow{0} ; M^{\prime}\left|B^{j} J^{k}(0)\right| \overrightarrow{0} ; M\right\rangle=2 \sum d \vec{p} d \vec{q}\left\{\psi_{M^{\prime}}^{*}\left(\vec{p} \mu_{1}^{\prime},-\vec{p} \mu_{2}\right) B^{j}\left(\vec{p} \mu_{1}^{\prime}, \vec{q} \mu_{2}^{\prime}\right)\right. \\
\left.-\psi_{M^{\prime}}^{*}\left(\vec{q} \mu_{1}^{\prime},-\vec{q} \mu_{2}^{\prime}\right) B^{j}\left(\vec{q} \mu_{1}^{\prime}, \vec{p} \mu_{2}\right)\right\} F_{\tau}^{k}\left(\vec{q} \mu_{2}^{\prime}, \vec{p} \mu_{1}\right) \psi_{M}\left(\vec{p} \mu_{1},-\vec{p} \mu_{2}\right)
\end{gathered}
$$

ii) to perform the integration in parts and necessary differentiations that gives the division $\mu_{d}=$ $\mu_{d}^{N R}+\mu_{d}^{R C}$ where

$$
\mu_{d}^{\mathrm{NR}}=\frac{2 m}{m_{d}} \mathcal{L} d \vec{p} \psi_{l^{\prime}}(p) \psi_{l}(p)\left\langle Y_{11}^{l^{\prime} 1}(\hat{\vec{p}})\left|\left[\mu_{n}+\mu_{p}\right] J^{z}-\left[\mu_{n}+\mu_{p}-\frac{1}{2}\right] L^{z}\right| Y_{11}^{l 1}(\hat{\vec{p}})\right\rangle,
$$




$$
\begin{gathered}
\mu_{d}^{\mathrm{RC}}=\frac{m}{m_{d}} \sum d \vec{p} \psi_{l^{\prime}}(p) \psi_{l}(p)\left(E_{\vec{p}}-m\right)\left[\frac{\mu_{n}+\mu_{p}}{E_{\vec{p}}}+\frac{1-\mu_{n}-\mu_{p}}{m}\right] \\
\left\langle Y_{11}^{l^{\prime} 1}(\hat{\vec{p}})\left|\hat{p}^{z}(\hat{\vec{p}} \vec{S})-S^{z}\right| Y_{11}^{l 1}(\hat{\vec{p}})\right\rangle . \\
\vec{L}=-i \vec{p} \times \frac{\partial}{\partial \vec{p}}, \vec{S}=\frac{1}{2}[\vec{\sigma}(1)+\vec{\sigma}(2)], \vec{J}=\vec{L}+\vec{S}
\end{gathered}
$$

iii) to utilize properties of the spin-angular functions $\left|Y_{1 M}^{l 1}(\hat{\vec{p}})\right\rangle$ deriving the well-known nonrelativistic form

$$
\mu_{d}^{\mathrm{NR}}=\left\{\mu_{n}+\mu_{p}-\frac{3}{2}\left[\mu_{n}+\mu_{p}-\frac{1}{2}\right] P_{D}\right\}\left[\frac{e}{2 m}\right]
$$

and the relativistic correction

$$
\begin{aligned}
\mu_{d}^{\mathrm{RC}} & =\frac{1}{3 \sqrt{2}} \int_{0}^{\infty} p^{2} d p\left(E_{\vec{p}}-m\right)\left[\frac{\mu_{n}+\mu_{p}}{E_{\vec{p}}}+\frac{1-\mu_{n}-\mu_{p}}{m}\right] \\
& \times\left\{\sqrt{2}\left[\psi_{2}^{2}(p)-\psi_{0}^{2}(p)\right]+\psi_{0}^{2}(p) \psi_{2}^{2}(p)\right\}\left[\frac{e}{2 m}\right] .
\end{aligned}
$$

Moreover, one can show that the deuteron quadrupole moment is expressed through the limit $\vec{q} \vec{D}(\vec{q})$ at $\vec{q} \rightarrow 0$. But for the present calculations we prefer to deal with

$$
Q_{d}=-\frac{1}{m_{d}^{2}}\left\{\left\langle\overrightarrow{0} ; 1\left|B_{z}^{2} J^{0}(0)\right| \overrightarrow{0} ; 1\right\rangle-\left\langle\overrightarrow{0} ; 0\left|B_{z}^{2} J^{0}(0)\right| \overrightarrow{0} ; 0\right\rangle\right\}
$$

After this, by proceeding likely steps i) - iii) we get

$$
Q_{d}=Q_{d}^{N R}+Q_{d}^{R C} .
$$

As in case of the magnetic moment we separate the "nonrelativistic" contribution

$$
Q_{d}^{N R}=\frac{1}{20} \int d q q^{2}\left\{2 \sqrt{2} \frac{d \psi_{0}(q)}{d q}\left(\frac{d \psi_{2}(q)}{d q}+3 \frac{\psi_{2}(q)}{q}\right)-\left(\frac{d \psi_{2}(q)}{d q}\right)^{2}-6 \frac{\psi_{2}^{2}(q)}{q^{2}}\right\}
$$

and the relativistic correction

$$
\begin{gathered}
Q_{d}^{R C}=\frac{1}{m_{d}^{2}} \sum_{i=1}^{4} I^{(i)} \\
I^{(1)}=-\frac{1}{5} \int d q q^{4}\left\{2 \sqrt{2} \frac{d \psi_{0}(q)}{d q}\left(\frac{d \psi_{2}(q)}{d q}+3 \frac{\psi_{2}(q)}{q}\right)-\left(\frac{d \psi_{2}(q)}{d q}\right)^{2}-6 \frac{\psi_{2}^{2}(q)}{q^{2}}\right\}, \\
I^{(2)}=-\frac{3 \sqrt{2}}{5} \int d q \frac{q^{3} E_{q}}{E_{q}+m}\left[\mu_{n}+\mu_{p}+\frac{E_{q}}{m}\left(\mu_{n}+\mu_{p}-1\right)\right] \\
\times\left\{\psi_{0}(q) \frac{d \psi_{2}(q)}{d q}-\psi_{2}(q) \frac{d \psi_{0}(q)}{d q}+3 \frac{\psi_{0}(q) \psi_{2}(q)}{q}+\sqrt{2} \frac{\psi_{2}^{2}(q)}{q}\right\} \\
I^{(3)}=\frac{1}{3} \int d q \frac{q^{4}}{\left(E_{q}+m\right)^{2}}\left[\mu_{n}+\mu_{p}-\frac{1}{2}+\frac{E_{q}}{m}\left(\mu_{n}+\mu_{p}-1\right)\right]
\end{gathered}
$$




$$
\begin{gathered}
\times\left\{4 \psi_{0}^{2}(q)+\frac{14 \sqrt{2}}{5} \psi_{0}(q) \psi_{2}(q)+\frac{37}{35} \psi_{2}^{2}(q)\right\} \\
I^{(4)}=\frac{4}{5} \int d q \frac{q^{4}}{E_{q}^{2}}\left\{2 \sqrt{2} \psi_{0}(q) \psi_{2}(q)-\psi_{2}^{2}(q)\right\}\left[2 E_{q}^{2}\left(\frac{d G_{E}^{p}(t)}{d t}+\frac{d G_{E}^{n}(t)}{d t}\right)\right. \\
\left.-\frac{q^{2}-10 E_{q} m-4 m^{2}}{4\left(E_{q}+m\right)^{2}}+\left(\mu_{p}+\mu_{n}\right) g_{1}(q)-\left(\mu_{p}+\mu_{n}-1\right) g_{2}(q)\right], \\
g_{1}(q)=\frac{q^{2}-2 E_{q} m}{\left(E_{q}+m\right)^{2}}, g_{2}(q)=\frac{q^{4}+3 q^{2} m^{2}+4 m E_{q} q^{2}+8 E_{q} m^{3}+4 m^{4}}{2 m^{2}\left(E_{q}+m\right)^{2}} .
\end{gathered}
$$

Recall that $\left.\frac{d G_{E}^{\tau}}{d t}\right|_{t=0}=\frac{1}{6}<r^{2}>_{\tau}^{c h}\left(t=\left(p^{\prime}-p\right)^{2}\right)$, where $<r^{2}>_{\tau}^{c h}$ is the charge r.m.s. radius of the proton or neutron.

\section{Several Numerical Results}

Following [ [8] (see Table 2 therein) the UCT row in Table 1 has been found with help of the least squares fitting of the on-energy-shell $R$-matrix elements, these solutions of the of inhomogeneous integral equations (66) [B]], to ones that are determined by eqs. (71)[B] with the potential $B$. In other words, it is the case where the theory is compared with the theory (not the experiment). One should note that the values of the adjustable parameters in the UCT row in Table 1 differ (sometimes considerably) from those in the UCT column of Table 2[8] since for describing the low-energy $n-p$ scattering and deuteron properties one has to extend the energy range of fitting.

\begin{tabular}{|c|c|c|c|c|c|c|c|}
\hline Model & Meson & $\pi$ & $\eta$ & $\rho$ & $\omega$ & $\delta$ & $\sigma, T=0(T=1)$ \\
\hline & $g^{2} / 4 \pi[f / g]$ & 14.4 & 3 & $0.9[6.1]$ & 24.5 & 2.488 & $18.3773(8.9437)$ \\
\hline Potential B & $\Lambda$ & 1700 & 1500 & 1850 & 1850 & 2000 & $2000(1900)$ \\
\hline & $m$ & 138.03 & 548.8 & 769 & 782.6 & 938 & $720(550)$ \\
\hline & $g^{2} / 4 \pi[f / g]$ & 13.395 & 5.0 & $1.2[6.1]$ & 17.349 & 5.0 & $22.015(5.514)$ \\
\hline UCT & $\Lambda$ & 2500 & 1219 & 1593 & 2494 & 2169 & $1200(2500)$ \\
\hline & $m$ & 138.03 & 548.8 & 769 & 782.6 & 938 & $720(550)$ \\
\hline
\end{tabular}

Table 1: The best-fit parameters for the two models. The row Bonn B (UCT) taken from Table A.1 in [24] (obtained by least squares fitting to reproduce the phase shifts and mixing parameters for the Bonn B potential). All the cutoff parameters $\Lambda$ are in $M e V$, and $n_{b}=1$ except $n_{\rho}=n_{\omega}=2$.

\begin{tabular}{|c|c|c|c|}
\hline Parameter & Bonn B & UCT & Experiment \\
\hline$a_{s}(\mathrm{fm})$ & -23.71 & -23.57 & $-23.748 \pm 0.010$ \\
$r_{s}(\mathrm{fm})$ & 2.71 & 2.65 & $2.75 \pm 0.05$ \\
$a_{t}(\mathrm{fm})$ & 5.426 & 5.44 & $5.419 \pm 0.007$ \\
$r_{t}(\mathrm{fm})$ & 1.761 & 1.79 & $1.754 \pm 0.008$ \\
\hline
\end{tabular}

Table 2: Low-energy parameters of the $n-p$ scattering. Experimental values are from Table 4.2 in [24]].

All our calculations have been done with the deuteron components obtained by solving the coupled equations (4.17) and depicted in fig. 1. Distinctions between the UCT and Bonn B curves result from the different behavior of the interactions at small distances. 


\begin{tabular}{|c|c|c|c|c|}
\hline & & \multicolumn{2}{|c|}{ UCT } & \\
\hline Parameter & Bonn B & NR & RC & Experiment \\
\hline$\varepsilon_{d}(\mathrm{MeV})$ & 2.22461 & \multicolumn{2}{|c|}{2.224} & 2.224575 \\
\hline$P_{D}(\%)$ & 4.99 & \multicolumn{2}{|c|}{49} & \\
\hline$\mu_{d}\left(e / 2 m_{p}\right)$ & 0.8516 & 0.8521 & $-8.981 \cdot 10^{-4}$ & $0.857406 \mp 000001$ \\
\hline$Q_{d}\left(\mathrm{fm}^{2}\right)$ & 0.2783 & 0.2972 & $3.115 \cdot 10^{-3}$ & $0.2860 \mp 0.0015$ \\
\hline
\end{tabular}

Table 3: Deuteron properties

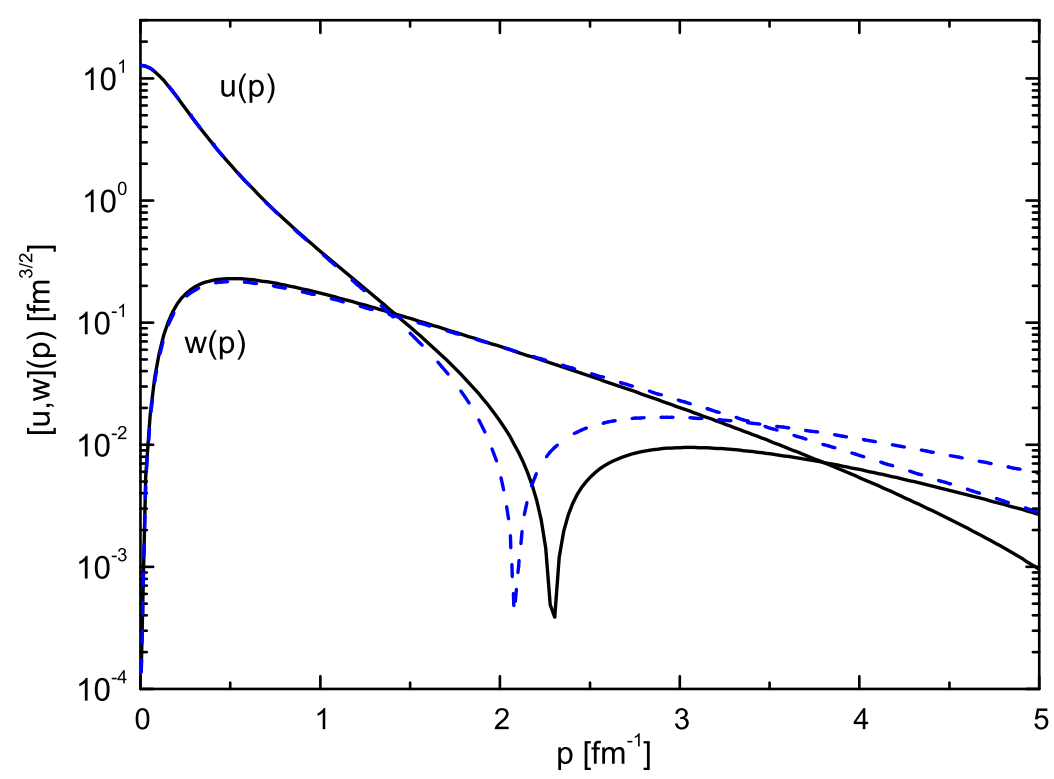

Figure 1: Deuteron wave functions $\psi_{0}^{d}(p)=u(p)$ and $\psi_{2}^{d}(p)=w(p)$. Solid ( dotted ) curves calculated with the Bonn B (UCT) potential.

\section{Summary}

One more application of the UCT method in the theory of nucleon-nucleon $(N-N)$ interaction has been presented. We have extended of our previous analysis [ []] of the $N-N$ scattering below the pion production threshold to treat the neutron-proton $(n-p)$ scattering at low energies and the deuteron static properties. Our calculations of deuteron magnetic and quadrupole moments have been carried out in the framework of a fresh description of EM interactions with nuclei (bound systems) using the clothed particle representation of the Hamiltonian, the boost and EM current density operators for the $n-p$ system. For this exposition we have seen a reasonable treatment of the low-energy $N-N$ scattering and deuteron properties. In the course of our current work we are trying to understand to what extent the deuteron quenching in flight affects the deuteron electromagnetic form factors.

In our opinion, the exposed approach has promising prospects, e.g., in the theory of decaying states (after evident refinements), certainly in quantum electrodynamics and, we believe, in quantum chromodynamics too. Such endeavors are under way. 


\section{References}

[1] A. Shebeko, M. Shirokov, Clothing procedure in relativistic quantum field theory and its applications to description of electromagnetic interactions with nuclei (bound systems), Progr. Part. Nucl. Phys. 44 (2000) 75

[2] A. V. Shebeko, M. I. Shirokov, Unitary transformations in quantum field theory and bound states, Phys. Part. Nuclei 32 (2001) 31 arXiv:nucl-th/0102037

[3] V. Korda, L. Canton, A. Shebeko, Relativistic interactions for the meson-two-nucleon system in the clothed-particle unitary representation, Ann. Phys. 322 (2007) 736 arXiv:nucl-th/0603025

[4] A. V. Shebeko, The method of unitary clothing transformations in quantum field theory: applications in the theory of nuclear forces and reactions, Chapter I in: Advances in Quantum Field Theory, ed.

S. Ketov, Intech 2012.

[5] E. Kazes, T. E. Feuchtwang, P. H. Cutler and H. Grotch, Gauge invariance and gauge independence of the S-matrix in nonrelativistic quantum mechanics and relativistic quantum field theories, Ann. Phys. 142 (1982) 80

[6] L. G. Levchuk, A. V. Shebeko, On a generalization of Siegert's theorem. A corrected result, Phys. At. Nuclei 56 (1993) 227

[7] L. Levchuk, L. Canton, A. Shebeko, Nuclear effects in positive pion electroproduction on the deuteron threshold, Eur. Phys. J. A 21 (2004) 29

[8] I. Dubovyk, A. Shebeko, The method of unitary clothing transformations in the theory of nucleon-nucleon scattering, Few-Body Syst. 48 (2010) 109 arXiv:1012.5406 [nucl-th]

[9] R. Machleidt, K. Holinde, C. Elster, The Bonn meson exchange model for the nucleon-nucleon interaction, Phys. Rep. 149 (1987) 1

[10] A. V. Shebeko, P. A. Frolov, A possible way for constructing the generators of the Poincaré group in quantum field theory, Few Body Syst. 52 (2012) 125 arXiv:1107.5877 [hep-th]

[11] P. L. Chung, B. D. Keister, F. Coester, Relativistic calculation of the deuteron quadrupole and magnetic moments, Phys. Rev. C 39 (1989) 1544

[12] F. M. Lev, E. Pace, G. Salme, Deuteron magnetic and quadrupole moments with a Poincaré covatiant current operator in the front-form dynamics, Phys. Rev. Lett. 83 (1999) 5250

[13] N. Honzawa, S. Ishida, Electromagnetic static moments of deuteron in the Bethe-Salpeter formalism, Phys. Rev. C 45 (1992) 47

[14] S. G. Bondarenko, V. V. Burov, A. V. Molochkov, G. I. Smirnov, H. Toki, The Bethe-Salpeter approach with the separable interaction for the deuteron, Prog. Part. Nucl. Phys. 48 (2002) 449

[15] K. Tamura, T. Niva, T. Sato, H. Ohtsubo, Relativistic effects on electron scattering of deuteron, Nucl. Phys. A 536 (1992) 236

[16] H. Arenhövel, F. Ritz, T. Wilbous, Elastic electron deuteron scattering with consistent meson exchange and relativistic contributions of leading order, Phys. Rev. C 61 (2000) 034002

[17] R. G. Sachs, High-energy behavior of nucleon electromagnetic form factors, Phys. Rev. 126 (1962) 2256

[18] J. L. Friar, S. Fallieros, Gauge-invariant nuclear Compton amplitude manifesting low-energy theorems, Phys. Rev. C 34 (1986) 2029 
[19] A. V. Shebeko, A generalization of Siegert's theorem and separation of center-of-mass, Sov. J. Nucl. Phys. 49 (1989) 30

[20] L. L. Foldy , Matrix elements for the nuclear photoeffect, Phys. Rev. 92 (1953) 178

[21] M. Garçon, J. W. Van Orden, The deuteron: structure and form factors, Adv. Nucl. Phys. 26, (2002) 293

[22] J. D. Jackson, L. D. Okun, Historical roots of gauge invatiance, Rev. Mod. Phys. 73 (2001) 663

[23] M. Rho, D. Wilkinson Mesons in Nuclei, North-Holland, Amsterdam 1979.

[24] R. Machleidt, The meson theory of nuclear forces and nuclear structure, Adv. Nucl. Phys. 19 (1989) 189 